\title{
Origin of room temperature ferromagnetic moment in Rh-rich [Rh/Fe] multilayer thin films
}

\author{
Dhishan Kande, ${ }^{1, a)}$ David Laughlin, ${ }^{1,2}$ and Jian-Gang Zhu ${ }^{1,2}$ \\ ${ }^{1}$ Materials Science and Engineering Department, Carnegie Mellon University, Pittsburgh, Pennsylvania \\ 15213, USA and Data Storage Systems Centre, Carnegie Mellon University, Pittsburgh, Pennsylvania 15213, \\ USA \\ ${ }^{2}$ Electrical and Computer Engineering, Carnegie Mellon University, Pittsburgh, Pennsylvania 15213, USA
}

(Presented 21 January 2010; received 31 October 2009; accepted 20 November 2009; published online 14 May 2010)

B2 ordered FeRh thin films switch from antiferromagnetic (AFM) to ferromagnetic (FM) state on heating above $350 \mathrm{~K}$ and switch back on cooling, with a hysteresis. This property makes FeRh a very attractive choice as a write-assist layer material for low temperature heat assisted magnetic recording (HAMR) media. Studies have shown that as we decrease the thickness of the FeRh films, the B2 phase is no longer AFM even below $350 \mathrm{~K}$ and there is a thickness dependant FM stabilization of the B2 phase. It was also proved that slightly Rh-richer compositions ( $>50$ at. \% Rh) were more preferable to stabilize the AFM phase. The current study focuses on growing highly ordered FeRh films by alternate layer rf sputtering of thin layers of iron and rhodium onto a heated substrate. It has been shown that films with rhodium content beyond 55 at. \% contain a disordered bcc FM phase which gives rise to residual moment at room temperature even for thicker films. (C) 2010 American Institute of Physics. [doi:10.1063/1.3360200]

\section{INTRODUCTION}

Bulk B2 FeRh undergoes a sharp transition from purely antiferromagnetic (AFM) to ferromagnetic (FM) state on heating above $350 \mathrm{~K}$ and the reverse transition occurs with a small hysteresis in temperature. ${ }^{1}$ This property makes this material a unique choice as a thermomagnetic switch for heat assisted magnetic recording (HAMR) media ${ }^{2}$ and magnetic random access memory (MRAM) applications. Early work on thin films of FeRh was done by electron beam melting on amorphous substrates ${ }^{3}$ but the low extent of ordering and the compositional fluctuations of these films resulted in broad magnetic transitions. More recently, sharp switching was demonstrated in sputtered films on glass substrates, with thicknesses above $200 \mathrm{~nm} .{ }^{4}$ Thinner films of this alloy with good switching have been fabricated by sputtering onto heated $\mathrm{MgO}$ (001) single crystal substrates and the exchange spring effect seen in $\mathrm{FePt} / \mathrm{FeRh}$ bilayers ${ }^{5}$ really led to increased interest in this material for HAMR applications. Cao et $a l .{ }^{6}$ studied the effect of postannealing on room temperature sputtered films and demonstrated that the best switching properties were for the films postannealed at $973 \mathrm{~K}$. Other studies $^{7}$ revealed that the composition had to be on the $\mathrm{Rh}$ rich side of 50-50 or else a two-phase mixture of the FM B2 phase and the AFM B2 phase is stabilized as indicated in the $\mathrm{Fe}-\mathrm{Rh}$ phase diagram. ${ }^{8}$

Here, we present an experimental study on the effect of excessive $\mathrm{Rh}$ on the FM B2 phase in B2 [Fe/Rh] multilayered film below the AFM-FM transition temperature. Alternating layer sputtering of the individual elemental layers is employed here to maximize the order parameter of a 001 oriented B2 structure.

${ }^{a)}$ Electronic mail: dkande@andrew.cmu.edu.

\section{EXPERIMENT}

FeRh films are fabricated by depositing Fe and Rh layers alternately onto $001 \mathrm{MgO}$ single crystal substrates heated to $803 \mathrm{~K}$. The rf sputtering was done at $15 \mathrm{~W}$ power and $5 \mathrm{mT}$ Ar pressure in the Leybold Z-400 system. To study the effect of thickness of the film, the composition of the alloy film was fixed by fixing the thickness ratio of Fe to Rh layers. Then the composition was varied to study its effect on the switching. X-ray diffraction (XRD) studies were done for all the films using the X'Pert Diffractometer. The magnetic measurements were performed on the Lakeshore Vibrating Sample Magnetometer. While doing the magnetic measurements, a constant in-plane field of $0.8 \mathrm{~T}$ was applied to saturate the film in the plane and moment versus temperature data was measured. In order to confirm that there was no clumping of the individual elements due to the alternating layer sputtering, x-ray photoelectron spectroscopy (XPS) was used to map out the composition depth profiles. The bulk compositions of the films were measured using the energy dispersive spectroscopy data from the XL-30 Scanning Electron Microscope. The thickness of the thin films was measured using the reflectivity set-up in the X'Pert Diffractometer.

\section{RESULTS AND DISCUSSION}

Figure 1 shows the XRD data of thin films of different Rh-rich compositions. It is clear that for Rh-richer films there is a rhodium-rich $\gamma$ fcc phase which decreases as we decrease the $\mathrm{Rh}$ content. Both the $\mathrm{B} 2$ phase and the $\gamma$ fcc phase grow textured on top of the $\mathrm{MgO}(001)$ substrate. Figure 2 shows the epitaxial relationships between the film phases and the substrate for the alloy film of composition 65 at. $\% \mathrm{Rh}$. The $110 \mathrm{~B} 2$ peaks are $90^{\circ}$ away from the nearest 


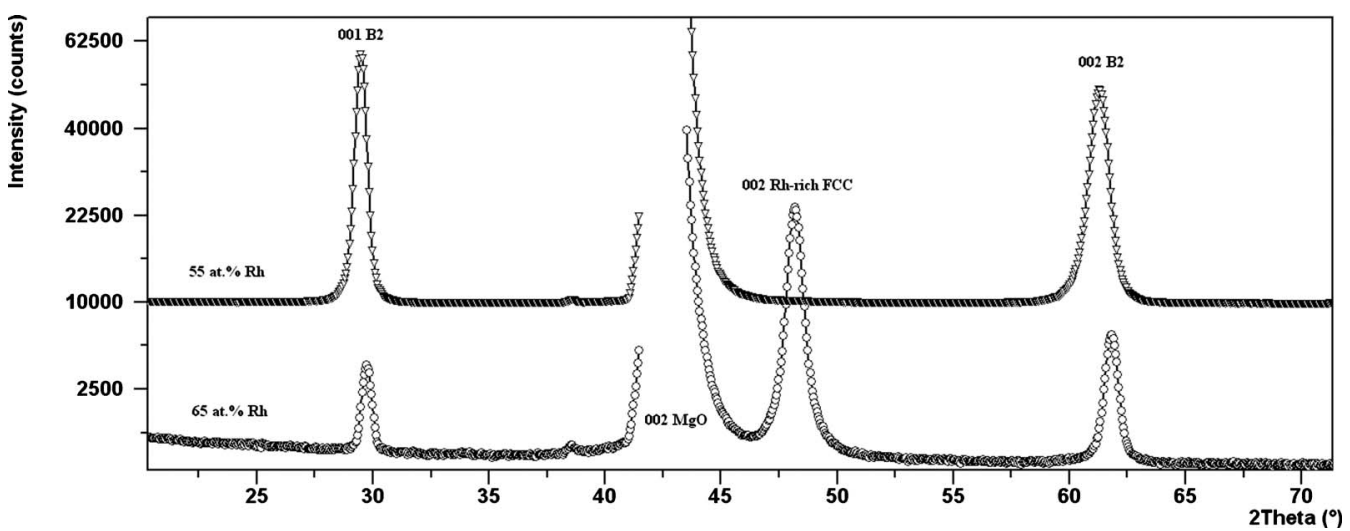

FIG. 1. Out of plane $2 \theta$ scans for the thin films of two different Rh-rich compositions.

$220 \mathrm{MgO}$ peaks meaning that the $001 \mathrm{~B} 2$ lattice plane is rotated by $45^{\circ}$ about the film normal to match perfectly with the single crystal $\mathrm{MgO}$ (001) plane. Figure 3 compares the magnetic measurements of two samples with the same composition of 65 at.\% Rh but different thicknesses of $45 \mathrm{~nm}$ and $20 \mathrm{~nm}$, respectively. For the thicker sample it was seen that there is a residual FM signal at room temperature, as shown in Fig. 3(a). On heating beyond $350 \mathrm{~K}$, an abrupt increase in the moment occurred due to the first order AFM to FM transition of the B2 phase. Subsequently the FM B2 phase became paramagnetic above $693 \mathrm{~K}$ but the moment did not go down to zero as expected for a pure AFM film. Further heating traced out the behavior of a FM phase with a Curie temperature of $1043 \mathrm{~K}$. From the equilibrium phase diagram the only phase with such a Curie temperature is the Fe-rich $\alpha$ bcc phase. But the XRD measurement did not show any peak splitting at the 002-peak position corresponding to the disordered phase, nor is there any other peak that is unaccounted for. Hence it is quite possible that the disordered phase has a composition very near to that of the B2 phase. Extrapolating the FM curve of the bcc phase to room temperature indicates that the FM moment is almost completely due to the bcc FM phase. This is not the case for the thinner film as shown in Fig. 3(b). For the thinner film there is again the same bcc FM phase but it does not contribute to all the room temperature FM moment. The additional FM moment is attributed to a
FM B2 phase. This is consistent with the literature where thickness dependent stabilization of the FM B2 phase has been reported. ${ }^{9}$ A theoretical study explaining such an observation has been given by Lounis et al. ${ }^{10}$ Since the transition is first order, the presence of B2 FM phase at room temperature means that the nuclei of the product phase are already present and hence the slope of the switching curve is not sharp as compared to the case of homogeneous nucleation of the product phase. This can be seen by comparing Figs. 3(a) and 3(b). Also it has been observed that the room temperature moment is not fully recovered after the cooling cycle but there is a kind of field pinning of the B2 FM phase. Similar effects have been indicated by Inoue et al. ${ }^{11}$ On decreasing the composition to 55 at. \% Rh, the signal from the bcc FM phase was reduced immensely, as shown in Fig. 4. This indicates that the presence of the $\gamma \mathrm{fcc}$ phase hinders ordering. Similar conclusions about the detrimental effect of the $\gamma \mathrm{fcc}$ phase were given by Ohtani and Hatakeyama ${ }^{12}$ This fact is supported by the integrated intensity ratios for the two samples. For the sample without the $\gamma$ fcc phase, I(001)/ $\mathrm{I}(002)$ was 0.76 and for the sample with the $\gamma$ fcc phase it was 0.45 .

In order to verify that the $\alpha$ bcc phase was not the result of sputtering alternate elemental layers, XPS studies were

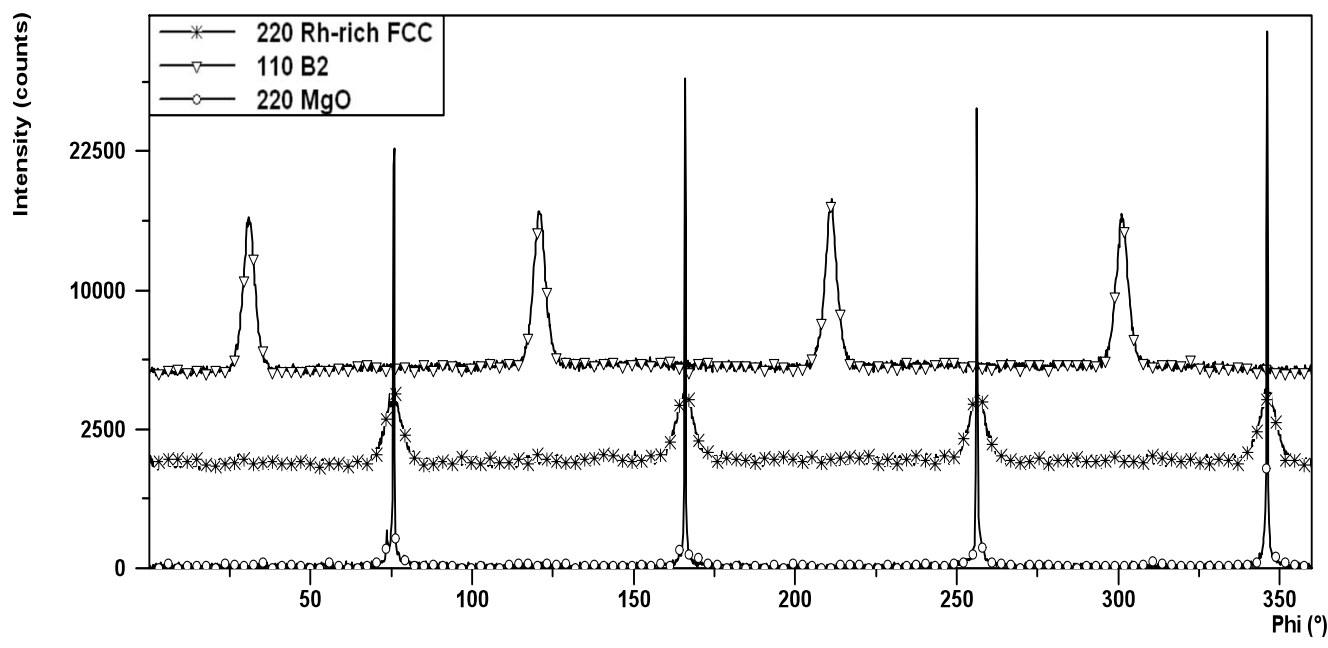

FIG. 2. $\phi$ Scans showing the epitaxial relationships in the $20 \mathrm{~nm}$ film of composition 65 at. $\%$ Rh. 


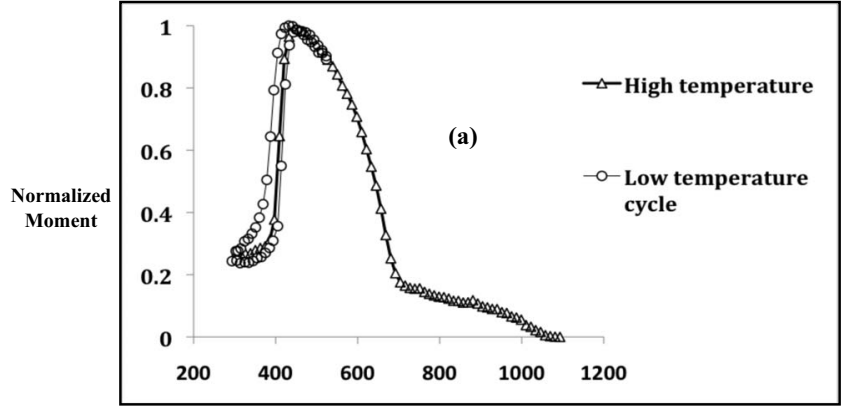

Temperature (K)

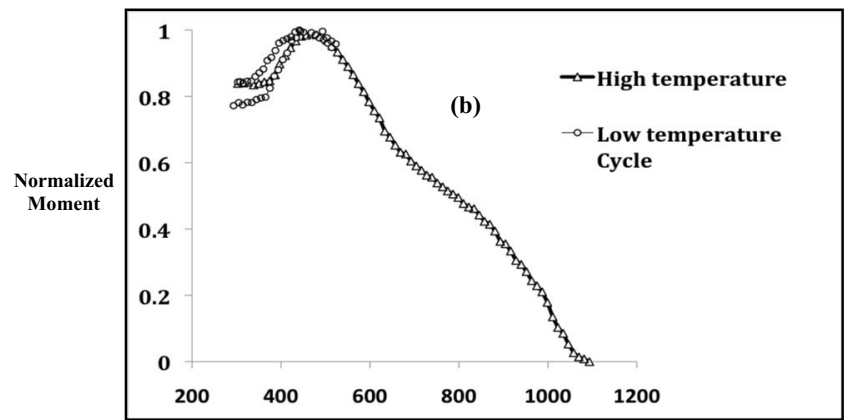

Temperature (K)

FIG. 3. (a) Magnetic measurements for the $45 \mathrm{~nm}$ film and (b) magnetic measurements for the $20 \mathrm{~nm}$ thin film.

done to get composition depth profiles. Figure 5 shows the XPS data for the $20 \mathrm{~nm}$ multilayered film. The composition is nearly homogeneous across the thickness of the film.

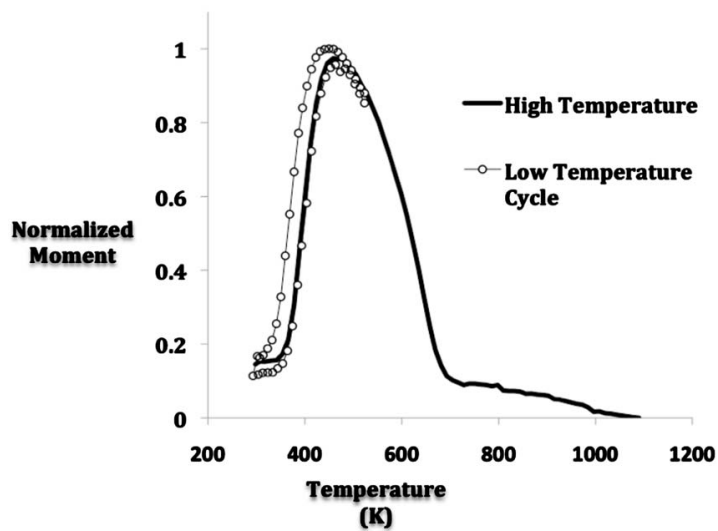

FIG. 4. Moment vs temperature measurements for the thin film of composition 55 at. \% $\mathrm{Rh}$ (40 nm thickness).

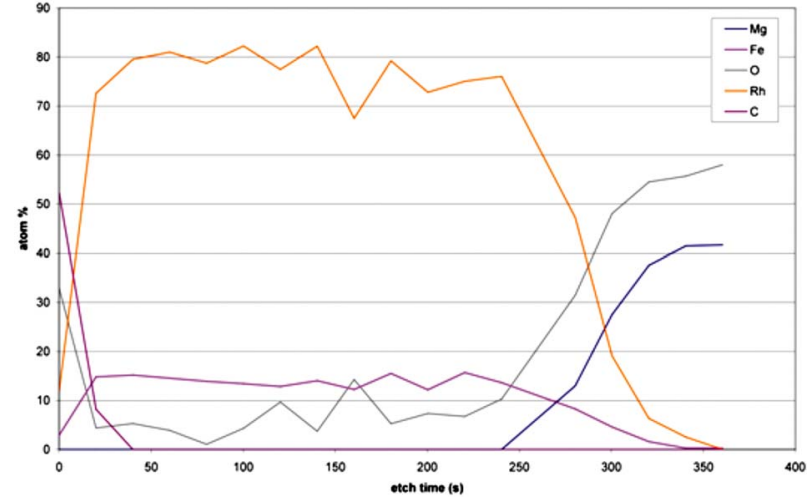

FIG. 5. (Color online) Composition depth profiles for $\mathrm{Fe}, \mathrm{Rh}, \mathrm{Mg}, \mathrm{O}$, and $\mathrm{C}$ from XPS measurement for the $20 \mathrm{~nm}$ film.

\section{SUMMARY}

It has been demonstrated that well-ordered B2 FeRh films can be fabricated by alternating layer sputtering of the individual elemental layers. For all the films studied there was a room temperature FM moment. For films thicker than $40 \mathrm{~nm}$, the room temperature FM moment was contributed by the disordered bcc phase only, whereas for the thinner films an additional component came from the B2 FM phase. This work indicates that the composition must be below 55 at. \% Rh to minimize room temperature FM moment from the bcc disordered phase. Accurate fitting of the moment versus temperature data can give quantitative information about the volume fractions of various magnetic phases and this work is currently under progress.

\section{ACKNOWLEDGMENTS}

We thank Dr. Paul Ohodnicki from PPG Industries, Pittsburgh for the XPS measurements and Data Storage Systems Center for supporting this research.

${ }^{1}$ J. S. Kouvel et al., J. Appl. Phys. 33, 1343 (1962).

${ }^{2}$ J.-U. Thiele et al., Appl. Phys. Lett. 82, 2859 (2003).

${ }^{3}$ J. M. Lommel, J. Appl. Phys. 37, 1483 (1966).

${ }^{4}$ S. Hashi et al., IEEE Trans. Magn. 40, 2784 (2004).

${ }^{5}$ J.-U. Thiele et al., IEEE Trans. Magn. 40, 2537 (2004).

${ }^{6}$ J. Cao et al., J. Appl. Phys. 103, 07F501 (2008).

${ }^{7}$ J. van Driel et al., J. Appl. Phys. 85, 1026 (1999).

${ }^{8}$ Binary Alloy Phase Diagrams, edited by T. B. Massalski (ASM International, Materials Park, OH, 1992), Vol. 2, p. 1760.

${ }^{9}$ I. Suzuki et al., J. Appl. Phys. 105, 07E501 (2009).

${ }^{10}$ S. Lounis et al., Phys. Rev. B 67, 094432 (2003).

${ }^{11}$ S. Inoue et al., J. Magn. Magn. Mater. 320, 3113 (2008).

${ }^{12}$ Y. Ohtani and H. Hatakeyama, J. Appl. Phys. 74, 3328 (1993). 